\title{
Assinatura Gravimétrica e Magnética da Bacia Sergipe-Alagoas
}

Sampaio, M.R.C.; LFNA-UFBA; Dutra,A.C.; DFTMA-UFBA

Copyright 2018, SBGf - Sociedade Brasileira de Geofísica

Este texto foi preparado para a apresentação no VIII Simpósio Brasileiro de Geofísica, Salinópolis, 18 a 20 de setembro de 2018. Seu conteúdo foi revisado pelo Comitê Técnico do VIII SimBGt mas não necessariamente representa a opinião da SBGf ou de seus associados. É proibida a reprodução total ou parcial deste material para propósitos comerciais sem prévia autorização da SBGt.

\section{Resumo}

This study has used gravimetric and magnetic data to search for the framework of the sedimentary sub-basin Sergipe and part of its basement. The main goals are to estimate parameters such as basement depth and vertical/lateral variation of the density in the domains of Estância, Canudos-Vaza-Barris, and in the section adjacent to Sergipe sub-basin, using geological and geophysical estrutures measured in rocks collected on the surface. Based on the information acquired we were able to process and interpret the gravimetric data troughout the basement adjacent region and its extension for the Sergipe sub-basin, so we could create a gravimetric modeling of the crustals domains and its tectonics relations. It was necessary to know the gravimetric anomalies and the density contrast on the surface basin to delineate the basement relief from Sergipe sub-basin. From those informations it was possible to estimate, besides the relief, the contrast value of the density on this sedimentary basin surface, being provided the gravimetric anomalie and the depth of the basement at some basin spots.

\section{Introdução}

A área de estudo pertence à bacia Sergipe-Alagoas, uma importante bacia sedimentar brasileira localizada na margem continental dos estados de Sergipe, Alagoas e uma parte de Pernambuco. Considerada a mais completa bacia em sucessões estratigráficas, esta apresenta um avançado potencial de exploração, e é limitada ao norte pela bacia de Pernambuco-Paraíba e ao sul pela Bacia de Jacuípe. Neste trabalho, o ponto central de estudo está na sub-bacia Sergipe, onde mais precisamente foi dado um enfoque á porção sul da mesma e á uma estrutura adjacente á sub bacia, anterior á sua formação conhecida como Domo de Itabaiana.

Através dos métodos geofísicos magnético e gravimétrico, os quais respectivamente utilizam dados medidos na região acerca do campo magnético e aceleração da gravidade no local constituímos esta pesquisa. Com tais dados geofísicos medidos é possível obter parâmetros de corpos em subsuperfície como a densidade e a susceptibilidade magnética, embora nesta pesquisa tais parâmetros já tenham sido medidos com base em estudos geológicos de superficie. Através do tratamento destes dados consegue-se não apenas estimar profundidades do embasamento como também delimitar extremos de estruturas, a exemplo do que ocorre com a utilização do artifício da Amplitude de Sinal Analítico (ASA) aplicado ao dado magnético. A grande vantagem dos dois métodos gravimétrico e magnético é a capacidade de recobrimento espacial com custos razoáveis, e são bastante utilizados para a detecção, localização e delineação de feições estruturais.

Como objetivo temos o delineamento dos limites do arcabouço sedimentar da sub-bacia Sergipe , a obtenção dos contrastes de densidade laterais e verticais nos domínios Estância, Canudos-vaza-barris e no trecho adjacente à mesma. Completando a pesquisa, objetivouse realizar a modelagem do Domo de Itabaiana, uma estrutura complexa de ortognaisses migmatíticos, inserida no domínio Vaza-barris da faixa de dobramentos sergipana, a qual já sofreu intensos processos tectônicos.

Através do processamento realizado, e as seguintes etapas de análises dos dados magnéticos e gravimétricos, e comparações com os dados geológicos da área encontraram-se os resultados apresentados.

\section{Metodologia/ Problema Investigado}

Neste trabalho a aquisição, processamento e interpretação de dados gravimétricos foram realizados para obtenção da densidade na subsuperfície da região do embasamento adjacente à Bacia Sergipe-Alagoas e além disso obter soluções de profundidade do embasamento da sub-bacia Sergipe, conhecendo as anomalias gravimétricas e o contraste de densidade na superfície da bacia. Correlacionamos os dados aeromagnéticos e gravimétricos a outros dados relevantes como propriedades físicas para definir unidades geológicas e seus limites.

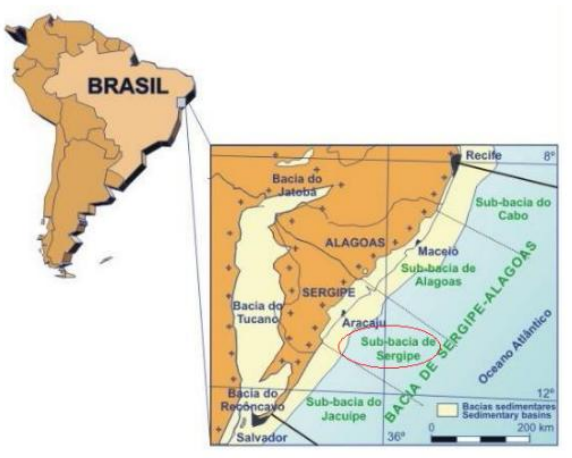

Figura 1 - Localização da sub-bacia Sergipe. 
Utiliza-se primeiramente a modelagem direta, cujo substrato foi representado por polígonos irregulares, de posição e tamanho conhecidos, mas com a propriedade física desconhecida. A modelagem de dados consistiu em construir um modelo de densidade em profundidade, calcular anomalia Bouguer para este modelo e comparar aos dados reais.

O segundo método utilizou a formulação não-linear no qual as fontes são representadas por prismas justapostos, com contraste da propriedade física conhecido, mas com a base da bacia a ser determinada. Nós utilizamos um método estável para interpretar a relevo do embasamento da sub-bacia sedimentar Sergipe. Este método delineia descontinuidades no relevo do embasamento.

\section{Dados Gravimétricos e Magnéticos}

Neste trabalho apresentamos os dados gravimétricos da bacia Sergipe-Alagoas e do embasamento a ela adjacente que obtivemos em campanhas do projeto Geoterm (IF-UFBA) e os dados que nos foram fornecidos pela Rede Nacional de Gravimetria através do banco de dados da ANP (Petrobras e outras empresas petrolíferas), da CPRM e de grupos de pesquisa. Além de dados fornecidos pelo Instituto Brasileiro de Geografia e Estatística (IBGE).

O levantamento gravimétrico consistiu na implantação de 10 bases gravimétricas de referência ao longo das linhas receptoras, sendo esta a poligonal que se obtém com a ligação de bases sucessivas, implantadas entre duas estações de referência e realizamos o levantamento de estações gravimétricas em várias campanhas entre 2013 a 2017.

Para esta transeção, contamos com o levantamento planialtimétrico necessário com GPS diferencial e as medidas gravimétricas com um gravímetro Scintrex CG5. Após a aquisição de dados gravimétricos segue-se com a etapa de processamento, conhecida como redução, que transforma as leituras do equipamento em acelerações e anomalias de gravidade para que sejam interpretadas na forma de mapas e perfis da anomalia Bouguer e separação regional residual (Blakely, 1996; Lowrie, 1997).

Com relação á sub-bacia foi aplicado o método de interpolação conhecido como krigagem através do software Oasis Montaj (Geosoft). Por conseguinte foi realizada a separação regional residual que consistiu na utilização de um algoritmo que através do método polinomial robusto (J.F. Beltrao, 1991) com grau 3 obtivemos o melhor resultado quanto á delimitação da bacia.

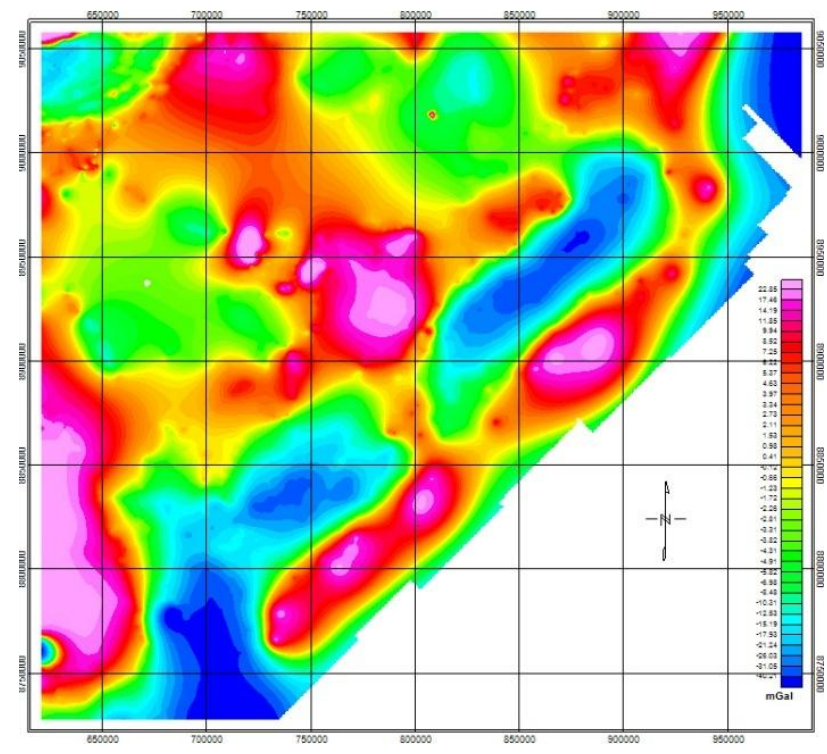

Figura 2 - Mapa resultante da região após a interpolação e separação regional residual.

Essa grande quantidade de dados gravimétricos foram utilizados para correlacionar esses dados a outros dados relevantes para definir unidades geológicas e seus limites, obter profundidades e mapear o contraste de densidade na crosta a partir da anomalia gravimétrica.

O Dado de magnetometria utilizado nesse trabalho pertence ao banco de dados da CPRM (Companhia de Pesquisa de Recursos Minerais). O dado consistiu do levantamento aeromagnético realizado na área de estudo e pertence ao projeto 1102_ESTADO_DE_SERGIPE. A aquisição de dados aeromagnéticos foi feita ao longo de linhas de voo paralelas e equiespaçadas. Para fins de mapeamento essas linhas foram voadas em direção N-S. As linhas de controle (tie-lines) foram voadas perpendicularmente às linhas de reconhecimento. $O$ espaçamento entre as linhas de vôo foi de $500 \mathrm{~m}$ na direção N-S e de $10 \mathrm{~km}$ entre as linhas de controle na direção E-W.

A anomalia magnética é apresentada por imagens coloridas com a intensidade em níveis de cores. Estes mapas forneceram boas informações quanto aos gradientes magnéticos, importantes para a determinação de profundidades e extensão de corpos.

Foram utilizados filtros no domínio da freqüência, com finalidade de realçar o sinal causado por fontes profundas que podem estar mascarados pela presença de sinal associado a fontes mais rasas. A Amplitude do sinal analítico (ASA) foi útil na delimitação de contatos entre feições magnéticas da área de estudo.

\section{Modelagem direta e inversão}

Para o campo magnético anômalo $\mathrm{g}\left(\mathrm{x}_{\mathrm{i}}, \mathrm{y}_{\mathrm{i}}, \mathrm{z}_{\mathrm{i}}\right)$ produzido pela distribuição de densidade $\left(\mathrm{p}_{\mathrm{j}}\right)$ o campo gravimétrico $g_{i}$ é produzido por fontes tomadas como prismas 
retangulares ou polígonos irregulares, dependendo do método adotado. As observações $g_{i}\left(x_{i}, y_{i}, z_{i}\right)$ são aproximadas por um funcional contínuo expressando a relação entre a propriedade física e as observações magnéticas correspondentes.

A modelagem gravimétrica é baseada no cálculo direto do campo gravitacional produzido por uma forma geométrica, no qual é construído um modelo inicial para o corpo fonte com base em informações geológicas e geofísicas disponíveis, de forma que a anomalia gerada por esse modelo se ajuste à anomalia gravimétrica observada. Este processo de ajuste de parâmetros é realizado até que as anomalias calculadas e observadas estejam o mais semelhantes possível (Blakely, 1996).

O método para modelagem utilizado neste trabalho é baseado no Método de Modelagem Bidimensional desenvolvido por (Talwani et al., 1959), onde as estruturas litológicas são desenhadas em forma de polígonos e são representados por per\#s ortogonais à direção da anomalia. O volume do corpo é obtido calculando-se a atração gravitacional causada por cada vértice do polígono modelado. Desta forma, quanto mais vértices o polígono tiver, melhor é a precisão do ajuste (dos Santos Silva, 2012). A anomalia calculada dependerá também do contraste de densidade utilizado entre os corpos do modelo, sendo a densidade constante para cada corpo.

Para inversão dos dados gravimétricos da sub-bacia Sergipe nós utilizamos um conjunto de observações gravimétricas produzidas por fontes arbitrárias, adotandose o sistema de coordenadas cartesianas. Assumimos que as fontes são corpos tridimensionais e incluímos a fonte em uma região que represente o substrato e que é discretizada em $M$ células. Dentro de cada célula assumimos que a propriedade física é constante. As observações foram aproximadas por um funcional contínuo expressando a relação entre a propriedade física e as observações geofísicas correspondentes; temos um conjunto de parâmetros associados a geometria e a posição de cada célula e p é um vetor dimensão $\mathrm{M}$ das propriedades físicas. Utilizamos a formulação não-linear no qual as fontes são representadas por prismas justapostos, com contraste da propriedade física conhecido, mas com topo ou a base a serem determinados segundo o método de inversão proposto por (Barbosa et al, 1997; Barbosa et al, 1999).

O modelo interpretativo consiste de uma grade de prismas retangulares verticais justapostos. Por simplificação, os centros dos prismas coincidem com os pontos de observação. Os parâmetros a serem determinados são as espessuras $p_{j}$ dos prismas. Detalhes do algoritmo podem ser obtidos em Barbosa et al. (1999).

\section{Resultados e Discussões}

Neste trabalho foram apresentados estimativas para parâmetros como espessura do embasamento e variação lateral e vertical da densidade nos domínios Estância, Canudos e Vaza-Barris no trecho adjacente à sub-bacia Sergipe.

Os modelos de densidade em profundidade obtidos a partir de dados gravimétricos residuais onde cada solução obtida possui um valor máximo de $\rho=2,98 \mathrm{~g} / \mathrm{cm}^{3}$. As principais estruturas regionais foram mapeadas em profundidade. Sugere-se a existência de intrusões do domo de Itabaiana, com profundidades que variam de 6 a $7 \mathrm{~km}$. Além disso estima-se para o domo uma densidade de $2,672 \mathrm{~g} / \mathrm{cm}^{3}$, apesar deste ser heterogêneo e apresentar uma série de rochas com diferentes densidades. Foi feita uma modelagem gravimétrica para um perfil SO/NE sobre o domo e tendo obtido a sua profundidade através da análise espectral foi percebido que esta estrutura é independente das adjacentes em subsuperfície, abrindo caminho para especulações acerca da sua história.

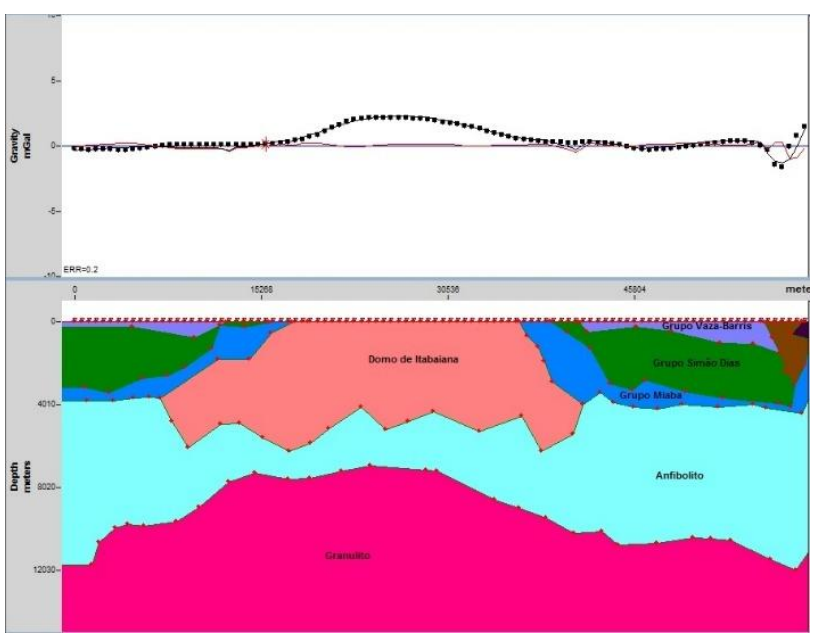

Figura 3 - Modelo do Domo de Itabaiana para um perfil SO/NE tirado do mapa gravimétrico da Figura 2

A partir do conhecimento da anomalia gravimétrica e o contraste de densidade na superfície da bacia em superfície, foi possível delinear o relevo do embasamento da Bacia Sergipe-Alagoas. A área da sub-bacia Sergipe apresentou valores máximos para espessura de $3,5 \mathrm{~km}$ de profundidade com 0 relevo do embasamento apresentando mudanças ocasionalmente abrutas. As anomalias gravimétricas foram invertidas com contraste de densidade de $\Delta \rho=-0,25 \mathrm{~g} / \mathrm{cm}^{3}$. O método de inversão se mostrou eficaz para se obter o delineamento do relevo do embasamento e as espessuras apresentam correspondência com as anomalias observadas.

As bacias sedimentares representam a principal fonte de concentração de petróleo. O estudo dessas bacias pode revelar a existência de petróleo em acumulações comercialmente exploráveis. Porém, a acumulação do petróleo depende da existência de armadilhas estruturais que o aprisionem e promovam sua concentração, 
somada à existência de matéria orgânica em quantidade, sua preservação e acumulação em profundidade. Por isso se torna importante apresentar resultados como o deste trabalho, que delineiam a interface entre 0 sedimento e embasamento da Bacia, e mapeiam estruturas do embasamento adjacente à bacia em profundidade.

O método utilizado permitiu o delineamento das descontinuidades no relevo do embasamento. A região em subsuperfície contendo a bacia foi discretizada em uma grade de prismas elementares justapostos, cujas espessuras estimadas representam a profundidade da interface de separação entre os sedimentos e o embasamento da sub-bacia Sergipe.

A inversão utilizou como critério de parada o erro do dado menor que $0,1 \%$. Os modelos de profundidades nas inversões apresentam correspondência com as anomalias observadas.

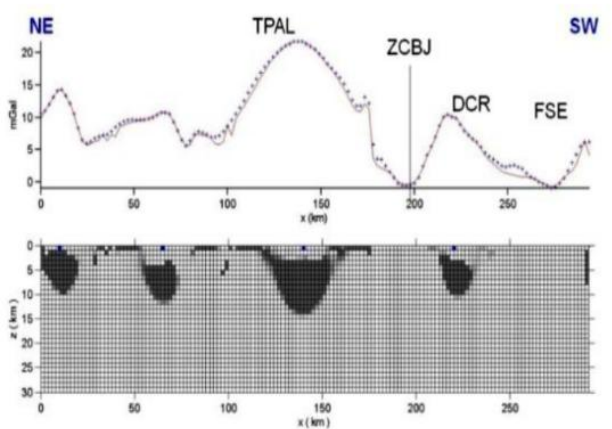

Figura 4- Soluções de contraste de densidade obtidas a partir da inversão de dados gravimétricos. Principais estruturas regionais: $Z C B J=$ Zona de Cisalhamento Belo Monte-Jeremoabo; TPAL = Terreno PernambucoAlagoas; $D R C=$ Domínio Rio Coruripe; FSE = Faixa Sergipana. Fonte: Dutra e Argollo (2017).

\section{Agradecimentos}

Agradecemos ao Promob - Cenpes - Petrobras pelo apoio através do projeto Petrobras 0050.0082543.13-9 e á CPRM e ANP pelos dados fornecidos.

\section{Referências}

Barbosa, V. C. F.; Silva, J. B. C.; Medeiros, W. E. 1997. Gravity inversion of basement relief using approximate equality constraints on depth. Geophysics, 62, 17451757.

Barbosa, V. C. F.; Silva, J. B. C.; Medeiros, W. E. 1999. Gravity inversion of a discontinuous relief stabilized by weighted smoothness constraints on depth. Geophysics, $64,1429-1438$.
Blakely, R.J., 1996, Potential Theory in Gravity and Magnetic Applications, Cambridge University Press, 441 pp.

dos Santos Silva, A. M. 2012. Aplicação de técnicas de modelagem direta e inversa para a caracterização gravimétrica da bacia de santos.

Lowrie, W., 1997, Fundamentals of Geophysics, Cambridge University Press, 354 pp.

Talwani, M.; Worzel, J. L. e Landisman, M. 1959. Rapid gravity computations for two-dimensional bodies with application to the mendocino submarine fracture zone, Journal of geophysical research,64 (1):49-59. 